\title{
Chapter 7 \\ Safety on the Italian Highways: Impacts of the Highway Chauffeur System
}

\author{
Serio Agriesti, Luca Studer, Paolo Gandini, Giovanna Marchionni, \\ Marco Ponti and Filippo Visintainer
}

\begin{abstract}
Road safety is certainly a main drive toward automated driving. Removing the human factor from driving maneuvers allows to prevent crashes caused or fostered by human flaws, such as distraction or tiredness. However, full automation on public roads is still a goal to be achieved, and different solutions are currently being developed. In the short term, one of the most promising examples is the Highway Chauffeur system, capable of performing fully automated driving in defined conditions and environments. The scope of this paper is to evaluate the number of crashes that could possibly be addressed through a defined market penetration of Highway Chauffeur vehicles (HC vehicles in the article). The evaluation is based on a bibliographical review and on an analysis of crash-related data recorded in Italy by the National Statistical Institute-ISTAT_-on public roads. By using the information publicly available on ISTAT's website, this paper provides a first magnitude concerning the number of crashes addressable by the Highway Chauffeur system, and final considerations on the future research to be carried out.
\end{abstract}

\subsection{Introduction}

The Highway Chauffeur (HC) system allows drivers to exit the driving loop and entrust the driving task to the vehicle, until some conditions are met. These conditions define the Operational Design Domain (ODD) of the system, namely, where and when the automated system is able to guarantee a safe and efficient driving. The elements generally defining an ODD are provided in [1], such as roadway typologies, geographical areas, speed ranges, environmental conditions, etc. A Highway Chauffeur system is intended to operate on highways, in a speed range of $0 \div 130 \mathrm{~km} / \mathrm{h}$ [2], as long as the equipped perceiving system [3] is not hindered by adverse weather

S. Agriesti $(\bowtie) \cdot$ L. Studer $\cdot$ P. Gandini $\cdot$ G. Marchionni $\cdot$ M. Ponti

Mobility and Transport Laboratory, Politecnico di Milano - Dipartimento di Design, Milan, Italy e-mail: serioangelo.agriesti@ polimi.it

F. Visintainer

Centro Ricerche FIAT, FCA EMEA Product Development, Safety and Driver Assistance Systems, Infotelematic Systems, Trento, Italy

X. Qu et al. (eds.), Smart Transportation Systems 2019, Smart Innovation,

Systems and Technologies 149, https://doi.org/10.1007/978-981-13-8683-1_7 
conditions such as heavy rain, snow, or fog. Moreover, in order to operate, the system requires well-defined lane markings and readable vertical signals; besides, it can benefit from information on the state of the road surface (e.g., wet surface, black ice). This information can be drawn both by dedicated sensors and through V2X communications [4]. As long as the system is engaged, the driving of an HC vehicle can be considered automated, with the driver completely out of the driving loop. Therefore, it is safer and more efficient than the driving of a human driver. Indeed, a sustainable deployment of the autonomous driving technology in future society can happen only if the technology itself reaches at least the same level of safety and efficiency currently achieved by a traditional vehicle. This aspect is essential for a safe and successful phase in of automated driving.

Taking into consideration the performance of the Highway Chauffeur system reported in literature, this paper explored the potential impact on safety of $\mathrm{HC}$ vehicles in the traffic flow of the Italian highways. This task was accomplished by analyzing crash data recorded by ISTAT in 2016, available at www4.istat.it/it/archivio/87539 [5]. The goal of the analysis presented in the paper is to define which crashes occurred in scenarios included in the most likely ODD of an $\mathrm{HC}$ vehicle, and which were caused or fostered by elements referable to automation. The approach of this study was based on the methodology presented in [6], even if with some expected differences, resulting from the adoption of two different databases for input data. In fact, [6] is a study conducted on the target crash population in the USA, while this paper considered the Italian framework on the basis of the data recorded by ISTAT. As a result of such analysis, this research provides an estimation of the percentages of potentially avoidable crashes for a $10 \%$ level of market penetration of the Highway Chauffeur with regard to the Italian highway network (according to [7], providing a forecast of the L3 market penetration in year 2025, $10 \%$ was considered a plausible value). This result aims to provide an overall estimation of the magnitude of the future impacts of the Highway Chauffeur system on the Italian highways. Indeed, the data analysis can be considered as an aggregate study and does not provide all the elements capable of retracing and characterizing every single crash event. Therefore, when the accident dynamics were uncertain, the accident was considered unavoidable and not addressable. This approach is conservative, and it probably underestimates the real impacts of the Highway Chauffeur system. Nonetheless, it meets the objective of providing a minimum baseline for potential safety impacts that will most likely be obtained in the medium term. In fact, the purpose of this paper is to identify crashes that can be addressed through the use of the Highway Chauffeur system, obtaining an overall value that represents both crashes potentially avoidable and crashes whose severity can be reduced. This kind of analysis is characteristic of an Ex-Ante phase, during which the new transport system is not yet implemented on roads but a first evaluation is needed to assess the magnitude of potential results and to have elements to design evaluation activities and field tests.

The paper is structured as follows: Section 7.2 provides an overview of the Highway Chauffeur's functioning and capabilities, defining the typical ODD. Section 7.3 presents the dataset employed in the analysis; it also analyses the most relevant fostering elements and causes connected to the recorded crashes when the dynam- 
ics resulted reasonably defined to evaluate the probable impact of automated HC vehicles (in analogous situations); lastly, it provides figures aimed at obtaining an impact assessment of the Highway Chauffeur system on safety. Section 7.4 provides comments on the results obtained and additional considerations on the assessment carried out. Finally, Sect. 7.5 presents the conclusions of the study and indicates future research directions, especially those considered useful to improve the estimation provided and make the magnitude more quantitative rather than indicative.

\subsection{Overview of the Highway Chauffeur System}

$\mathrm{HC}$ vehicles can perform both overtaking and lane changing [8-10] with different levels of aggressiveness [11, 12]. Moreover, when facing congestion, the Traffic Jam Chauffeur function guarantees a safe and efficient driving during the Stop\&Go regime. To allow the driver to engage secondary tasks, the Highway Chauffeur system exploits onboard equipment that includes Adaptive Cruise Control (ACC), Lane Keeping Assistant, and Automated Braking System, just to mention a few. An essential role is thus played by the software and hardware components [3]. With regard to the longitudinal control, while in automated mode, the $\mathrm{HC}$ vehicle relies on the ACC to keep a cruise speed value or a safe distance/time gap from the vehicle in front of it. To accomplish this task, long- and short-range radars onboard allow the system to compute the safe and desired time gap on the basis of the speed difference and the distance between the $\mathrm{HC}$ vehicle and the one in front of it. At the same time, the perceiving system reacts faster than drivers to the braking of the vehicle in front of it, bypassing almost completely the human perception and reaction time [13]. Moreover, giving the automated system a longitudinal control ensures that the acceleration and deceleration regimes do not suffer from the driver's tiredness or distraction (typical of human drivers), thus reducing the number of harsh braking and the possible resulting collisions.

As mentioned, the HC system can also accomplish lane keeping, lane change, and overtaking without the need to re-engage the human driver. To manage this task, the perceiving system employs the onboard sensors to measure both the available spaces and the driving speed on the target lane, deriving safe time gaps to perform the maneuver. In this paper, the assumption is that the HC vehicle is capable of identifying the available gaps on the other lane and to act accordingly.

\subsubsection{Approach}

Based on the literature about the HC system, it is possible to analyze crash-related data to determine what element caused or fostered crashes and if these occurred in scenarios ascribable to the typical ODD. Therefore, if the triggering or fostering factor is addressable by automated driving, a first evaluation of the number of avoidable 
or mitigated crashes is provided. These crashes can be defined as Target crashes (as in [14]) representing "the maximum potential safety benefit if the fully deployed system was $100 \%$ effective in reducing target crashes." An example referring to the HC system is a crash caused by the driver's distraction, occurred on a highway, in a section with clear lane marking and signaling, dry pavement and fair weather. An hypothesis imposed by the features of the data available concerned weather conditions: ISTAT's public database does not consider different levels of severity for adverse weather conditions such as, for example, rain. Thus, the only choice was to consider each of these events out of the ODD system (that, actually, could endure light rain). The same consideration was made for snow, fog, and hail. Thus, the accidental events occurred clearly within the ODD's boundaries were analyzed, in order to give a more realistic estimation. A similar approach was followed in [14], investigating V2I communications: "Targeted crashes are crashes that could potentially be eliminated through the deployment of a specific V2I application or set of applications (i.e., researchers should determine the potential benefit of an application area, assuming 100\% effectiveness and 100\% deployment)." It should also be highlighted how the analyses carried out in [14] are based on the identification of pre-crash scenarios, similarl to what was accomplished in this paper. Another research, within the activities of the U.S. Department of Transportation and adopting a similar approach, is provided in [15], designed with the objective to estimate "the upper limit of annual police-reported crashes that could potentially be addressed with IntelliDrive safety systems based on vehicle-to-vehicle communications or vehicleto-infrastructure cooperation." Even in this work, the result is a magnitude rather than a figure, as in this paper. This choice seems to be forced by the limited number of field operational tests on public roads characterizing both cooperative and autonomous vehicles. Moreover, it can be often difficult to assess the actual impacts on safety also through the Field Operational Test, due to the limited number of actual crashes. Therefore, this paper should be considered as a first tool for future evaluations carried out by road authorities, safety organizations, or researchers and evaluators. Indeed, it provides a first step useful to highlight which crashes are most likely preventable or reducible in severity thanks to an automated or semi-automated vehicle.

In the framework of road safety theories, the presented approach finds itself within the "Causal accident theory" category and as an accident causation approach tries mostly to consider the direct in-vehicle modification of the driving task [16]. The direct in-vehicle modification is indeed the main safety mechanism triggered by the $\mathrm{HC}$ system. Of the other safety mechanisms listed in [16], the following analysis considers also the modification of accident consequences while some relevant subjects are highlighted as future research topics but not included in the analysis (mostly due to the limits of the literature and of crash-related data on Italian roads). Of these safety mechanisms, the ones that will be mostly influenced by a certain market penetration of L3 vehicles are as follows:

- The indirect modification of user behavior. This mechanism concerns mostly the Take-Over maneuver during which the human driver takes the control of the vehicle 
back. That maneuver falls outside the set of crashes analyzed in Sect. 7.3 and thus doesn't affect the overall analysis.

- The long-term behavioral adaptation of the human driver. This mechanism should impact mostly crashes outside the considered ODD. Still, preferences about the chosen time gap or the acceleration regime of the L3 vehicle (when engaged) are questions that don't find a univocal answer in the literature.

- Modification of road user exposure and of modal choice. These two mechanisms are the ones that will probably affect the most the presented analysis. It is clear that a certain level of crash reduction can be outclassed by a relevant increase of kilometers driven indeed (due to the reduced cost of travel time, while the vehicle drives itself and the human driver is allowed to do something else). The analysis reported in this paper shouldn't be relevantly affected by an increase of road user exposure due to the short time horizon and the low market penetration considered. In fact, the changes of the travel patterns will probably arise in the medium-long term and currently impossible to quantify.

\subsubsection{Equipment Overview and Effectiveness of the System}

In order to bound the capabilities of the hypothesized HC system to the analysis carried out in paragraph 3, a short overview of the equipment of an L3 vehicle is provided. Moreover, as in [17], each one of the following components is related to the kind of crash that it addresses. Also, it should be noted that some of the crashes are not addressable by one single component but can be addressed by the system as a whole (through data fusion and software processing).

An L3 vehicle on the market by the year 2025 is considered equipped at least of Improved Advanced Cruise Control [18], Lane Keeping Assist, Automated Braking System, Front Collision Warning, Lane Change Assistance, and Blind Spot Detection System. All these components can be related to addressable crash types, as, for example, in [17]. For the present analysis, the following clusters are considered:

- Improved Advanced Cruise Control + Forward Collision Warning + Automated Braking System: head crash, lateral-head crash (within the cut-in scenario), rear end, and collision against an obstacle.

- Lane Keeping Assist + Lane Change Assistance + Blind Spot Detection System: lateral crash, road departure, and collision against an obstacle.

As mentioned, these single components can usually address the corresponding crashes with a level of effectiveness or eventually through the driver intervention (that is also characterized by a certain effectiveness). For an L3 vehicle, additional elements should be considered though, namely, onboard sensors, data fusion, and software processing. Through these elements, the hypothesis of $100 \%$ effectiveness can be adopted without compromising the results of the analysis. In fact, the first relevant difference between an automated system and a human driver is the perception capability. The automated system drives the vehicle mainly thanks to the sensors suite 
and the software. The minimum equipment in this case is composed of long- and short-range radars, LIDAR, and cameras. Each of these components has strengths and weaknesses, mostly covered by the other onboard sensors as explained in [19]. The hypothesis in this case is that, by 2025 , the sensor suite is going to correctly perceive the surroundings of the vehicle with no system failures, as long as the ODD conditions are met, reaching a full awareness. This is the first, relevant, difference between an L3 system and a human driver and is expected to influence all the crashes (analyzed in Sect. 7.3) in which an incorrect perception of the surroundings presumably took an important role in the crash. Through the perception process, once each sensor recorded information, the software is in charge to process all the inputs and to define a trajectory. This is the second, relevant, advantage of the $\mathrm{HC}$ on a human driver because the software does not suffer from tiredness or distraction, it is not indecisive and it can keep up the same level of performance even after hours of driving. As it will be deepened, these factors contribute to a really high percentage of crashes. Moreover, [17] is a work that summarizes the effectiveness of Driving Assistance systems and Connected Vehicle technologies on the basis of a bibliographical review.

Another work that achieves a similar task is [20], from which another valuable insight on the relationship between pre-crash scenarios and driving assistance system was obtained. Moreover, also in this work, a set of coefficients expressing the effectiveness of these technologies in addressing crashes was provided.

Unfortunately, after careful consideration it was decided to not use the coefficients arising from $[17,20]$ in this paper, mainly because all the coefficients refer to L0 or to L1 systems and quantify the ability of a single component to address a certain pre-crash scenario (this reflects also the current state of the art and the available bibliography). This means that the contributions of the sensors' suite and of the onboard software are ignored, even though they are the ones that nullify fostering factors such as distraction or tiredness in the pre-crash scenarios. Thus, it would be extremely conservative and also incorrect to apply these coefficients to a 2025 L3 vehicle, even by combining the contributions of all the technological components onboard. This imposes the hypothesis of $100 \%$ effectiveness, which is a limitation difficult to overcome only with the current literature. Moreover, the amount of field tests currently carried out in Europe is still limited and won't provide enough data to improve this type of analysis on a crash database. Thus, the need for surrogate methodologies arises and this kind of safety assessment could strongly benefit from analyses such as the one carried out in Sect. 7.3.

\subsection{ISTAT's Database-2016 Microdata}

ISTAT is the Italian National Statistical Institute that provides, among others, public data on road accidents involving injured or dead people. The macro-data for public use concerning the entire national network was considered for a first evaluation of crash scenarios potentially addressable by the Highway Chauffeur system when the driving task is entrusted to automation. Therefore, an analysis was conducted on the 
crashes that occurred in 2016 on the Italian public roads. The dataset used includes the following details (only the relevant ones are listed below):

"Autonomous vehicle based systems are also considered to evaluate what additional safety enhancements they can effect. (...). Applicable crashes include rearend crashes, lane departures, lane change or merge crashes, curve speed or excessive speeding crashes, and stop sign violations. It is assumed that AV systems could potentially address pedestrian, cyclist, and animal crashes as well as loss of control, road departure, and maneuver crashes in which speeding is a contributing factor" [15].

A first selection was carried out among all the crashes that occurred considering only the crashes occurred on highways (amounting to 9,360 in 2016), since the HC function can only prevent accidents on the type of road for which it is designed. The research then considered only the events (amounting to 6,408 crashes in 2016, $69 \%$ of the 9,360 occurred) that presumably occurred in domains included in the typical ODD (as derived from literature). On the basis of the data available, this first selection considered the features listed in Fig. 7.1: paved road surfaces, dry roads, both vertical and horizontal signals, and clear weather conditions. Considering the resulting set of crashes, evaluations were made concerning the circumstances that fostered or caused the events in order to obtain the number of accidents that could have been prevented or mitigated in severity if one of the two vehicles had been driven automatically (Fig. 7.2). It should be mentioned that the $\mathrm{HC}$ vehicle was considered capable of passing through roadworks [21], thus the temporary signaling was not considered sufficient to discard this dataset (equal to around 1\%). The details on the circumstances considered as cause or contribution to the crash occurrence are presented below.

Distracted driving or indecisive behavior. When the HC system is engaged, it continuously scans the environment and the surrounding road users, constantly uploading the relevant information without getting tired or distracted. Moreover, as long as the HC vehicle finds itself in its ODD, no undecided behavior should arise. In fact, an automated system decides in a short timeframe, almost instantaneously. Exceptions to this assumption can concern system failures, which are not considered in this paper (being extremely bound to the control algorithms of each OEMs and also rare enough not to impact the evaluation). Considering all the crashes that occurred in the specified ODD, 1,291 were caused or fostered by distracted driving or an indecisive behavior. Potentially, they all appear possibly avoidable thanks to the HC system. From an in-depth analysis, it appears that

- 839 crashes occurred with no additional cause or fostering element ascribable to the other vehicle involved. Thus, they are all addressable by an $\mathrm{HC}$ vehicle and can also be considered avoidable, considering that the human flaw would be neutralized. 623 of these events were rear-end collisions which an HC system should be able to address and very likely prevent.

- 199 collisions occurred while both drivers involved were distracted or showed an indecisive behavior. 144 of these were rear-end collisions, thus probably avoidable when the $\mathrm{HC}$ vehicle is the one behind. With the data available, it cannot be reasonably determined what would happen if the $\mathrm{HC}$ vehicle were the one in 


\begin{tabular}{|c|c|c|}
\hline CVADA Technology & Automation Level(SAE) & Target Pre-Crash Type and Pre-Cash Scenarios \\
\hline NS: Forward Collision Waming (FCW) & 0 & Rear-End: \\
\hline NS: Autonomous Emergency Braking (AEB) & 1 & 1 Lead Vehicle Stopped \\
\hline NS: Collision Warning System (CWS) & 0 & 2 Following Vehide Making a Maneuver \\
\hline S: Forward Collision Warning (FCW) + Adaptive Cruise Control(ACC) & 1 & 3 Lead Vehicle Decelerating \\
\hline S: Forward Collision Warning (FCW) + Autobrake & 1 & 4 Lead Vehicle Moving at Lower Constant Speed \\
\hline S: Forward Collision Warning (FCW) + Autonomous Emergency Braking (AFB) & 1 & 5 Lead Vehicle Accelerating \\
\hline S: Adaptive Cruise Control(ACC) + Advanced Braking System (AdvBS) & 1 & \\
\hline $\begin{array}{l}\text { S: Adaptive Cruise Control(ACC) + Advanoed Braking System (AdvBS) + Collision } \\
\text { Warning System (CWS) }\end{array}$ & 1 & \\
\hline S: Collision Mitigation Brake System (CMBS) & 1 & \\
\hline NS: Pedestrian Crash Avoidance and Mitigation System(PCAM) & 1 & Pedestrian: \\
\hline & & $\begin{array}{l}1 \text { Pedestrian Crash With Prior Vehicle Maneuver } \\
2 \text { Pedestrian Crash Without Prior Vehicle Maneuver }\end{array}$ \\
\hline NS: Blind Spot Warning (BSW) & 0 & Lane Change: \\
\hline NS: lane Change Warning (LCW) & 0 & 1 Vehicle(s) Turning - Same Direction \\
\hline s: Blind Spot Waming (BSW) + Lane Change Warning (LCW) & 0 & $\begin{array}{l}2 \text { Vehicle(s) Changing Lanes - Same Direction } \\
3 \text { Vehicle(s) Drifting - Same Direction }\end{array}$ \\
\hline NS: Intersection Movement Assist (MM/) & 0 & $\begin{array}{l}\text { Crossing Paths: } \\
\text { - Vehicle Tuming Right at Signalized Junctions } \\
\text { - Vehicle Tuming at Non-Signalized Junctions } \\
\text { - Straight Crossing Paths at Non-Signal ized Junctions } \\
\text { - Running Stop Sign } \\
\text { - Running Red Light }\end{array}$ \\
\hline NS-CV: Left Turn Assis(LTA) & 0 & Crossing Paths: \\
\hline S. Collision Mitigation Brake System (CMBS) & 1 & $\begin{array}{l}\text { - Left Turn Across Path from Opposite Directions at Nca. } \\
\text { - Signalized Junctions } \\
\text { - Left Turn Across Path from Opposite Directions at Signalized } \\
\text { Junctions }\end{array}$ \\
\hline NS: Lane Departure Warning(LW) & 0 & Run.Off-Roast: \\
\hline S. Lane Departure Warning(LDW) + Curve Speed Waming(CSW) & 0 & $\begin{array}{l}\text { - Road Edge Departure With Prior Vehicle Maneuver } \\
\text { - Road Edge Departure Without Prior Vehicle Maneuver } \\
\text { - Road Edge Departure While Backing Up }\end{array}$ \\
\hline NS: Electronic Stability Control (ESC) & 1 & $\begin{array}{l}\text { Run-Off-Road: } \\
\text { - Control Loss without Prior Vehicle Action } \\
\text { - Control Loss with Prior Vehicle Action }\end{array}$ \\
\hline NS: Backup Collision Intervention (BCD & 1 & Badking: \\
\hline NS: Rearview Cameras (RCA) & 0 & - Backing Up into Another Vehide \\
\hline
\end{tabular}

Note:S stands for the integrated system, while NS stands for non-syste.

Fig. 7.1 Tested CV\&DA technologies and corresponding pre-crash scenarios [17]

\begin{tabular}{|c|c|c|c|}
\hline Road Type & \multicolumn{3}{|c|}{ According to the Italian classification } \\
\hline Road Section & $\begin{array}{l}\text { - Crossing } \\
\text { - Roundabout }\end{array}$ & $\begin{array}{l}\text { - Intersection } \\
\text { - Straight Road }\end{array}$ & - Curve \\
\hline Road Surface & $\begin{array}{l}\text { - Paved } \\
\text { - Unpaved }\end{array}$ & \multicolumn{2}{|c|}{ - Paved-Dismaled } \\
\hline Surface State & $\begin{array}{l}\text { - Dry } \\
\text { - Wet }\end{array}$ & $\begin{array}{l}\text { - Slippery } \\
\text { - Frozen }\end{array}$ & - Snow \\
\hline Signage & $\begin{array}{ll}\text { - Absent } \\
\text { - Vertical }\end{array}$ & - Orizzontal & $\begin{array}{l}\text { - Both vertical and } \\
\text { horizontal }\end{array}$ \\
\hline Weather Condition & $\begin{array}{l}\text { - Clear } \\
\text { - Fog }\end{array}$ & $\begin{array}{l}\text { - Rain } \\
\text { - Hail }\end{array}$ & - Snow \\
\hline Kind of crash & $\begin{array}{l}\text { - Rear-end collision } \\
\text { - Head-on collision }\end{array}$ & $\begin{array}{l}\text { - Lateral collision } \\
\text { - Skid }\end{array}$ & - Etc. \\
\hline $\begin{array}{l}\text { Kind of vehicle } \\
\text { involved }\end{array}$ & $\begin{array}{ll}\text { - } & \text { Car } \\
\text { - Emergency }\end{array}$ & $\begin{array}{l}\text { vehicle } \\
\text { Truck }\end{array}$ & $\begin{array}{l}\text { - Bus } \\
\text { - Motorcycle }\end{array}$ \\
\hline $\begin{array}{l}\text { Circumstance that } \\
\text { fostered the event }\end{array}$ & & eported in the analy & \\
\hline
\end{tabular}

Fig. 7.2 ISTAT's relevant data—in bold the data considered for the analysis 
front, though it can be reasonably asserted that an $\mathrm{HC}$ vehicle performs a shorter and smoother braking. Nevertheless, it cannot be assessed in how many of these accidents the driver of the vehicle behind was so distracted or indecisive that the rear-ended crash would have occurred regardless of the harshness of the braking of the vehicle in front. Therefore, these 144 events were not considered among the ones addressable by an $\mathrm{HC}$ vehicle. 13 collisions were lateral-frontal crashes potentially avoidable if the $\mathrm{HC}$ vehicle performed the lane-changing maneuver, adequately scanning the other lane as mentioned in Sect. 7.2. They can be considered addressable also when the $\mathrm{HC}$ vehicle is the one behind: even if the preceding vehicle performs a risky lane change, the $\mathrm{HC}$ vehicle should be alerted and ready to decelerate. This consideration is based on the assumption that the ACC of the $\mathrm{HC}$ vehicle receives more information through the enhanced perceiving system of an L3 vehicle and thus reacts faster and better than a standard ACC [18].

- 22 collisions occurred because the other vehicle involved did not keep a safe distance from the one in front of it. This kind of crash is most likely addressable because the $\mathrm{HC}$ vehicle keeps a safe distance from the one in front and avoids harsh braking, thus lowering the chances of the vehicle behind to rear end.

- In seven cases, the other vehicles involved were driving at a speed higher than the speed limit. In six of these cases, the accident resulted in a rear-end collision. It should be noted that the $\mathrm{HC}$ vehicle brakes in a smoother way compared to a possibly distracted human driver, making a rear end less likely. Since the dynamics of the crash are not clear, these cases were not considered among the ones addressable.

- 35 collisions took place against an obstacle on the road, five of which during a turn. It should be noted that the HC system should be able to detect this obstacle while in its ODD and prevent the collision. Such collisions are thus addressable by the $\mathrm{HC}$ system.

In conclusion, out of the 1,291 collisions caused or fostered by distraction or an indecisive behavior, 1,136 (around 88\%) resulted presumably addressable by the L3 system if at least one of the two vehicles involved is an $\mathrm{HC}$ vehicle. The number of crashes addressable in such case is equal to around $18 \%$ of the 6,408 included in the ODD. It should also be highlighted that, in the bullet list presented above, only some examples were reported and commented, while the same analysis was carried out for each one of the 1,291 scenarios recorded in ISTAT's database (Fig. 7.3).

Insufficient safety distance. As mentioned in Sect. 7.2, an $\mathrm{HC}$ vehicle driving with the system engaged relies on the ACC for a longitudinal control. This means that a safe time gap is always kept between the $\mathrm{HC}$ vehicle and the one in front of it. Therefore, the contribution of the safety distance to the crash event is removed from the equation. Considering only the ODD, 1,679 accidents were caused by an insufficient safety distance. From an in-depth analysis, it appears that

- 1,570 of the crashes occurred were not fostered by flaws ascribable to the other vehicles involved. Therefore, they are all considered addressable by the HC system and, thus, likely preventable. 1,285 of these events where rear-end collisions, which a Highway Chauffeur system should be able to address and very likely prevent. 


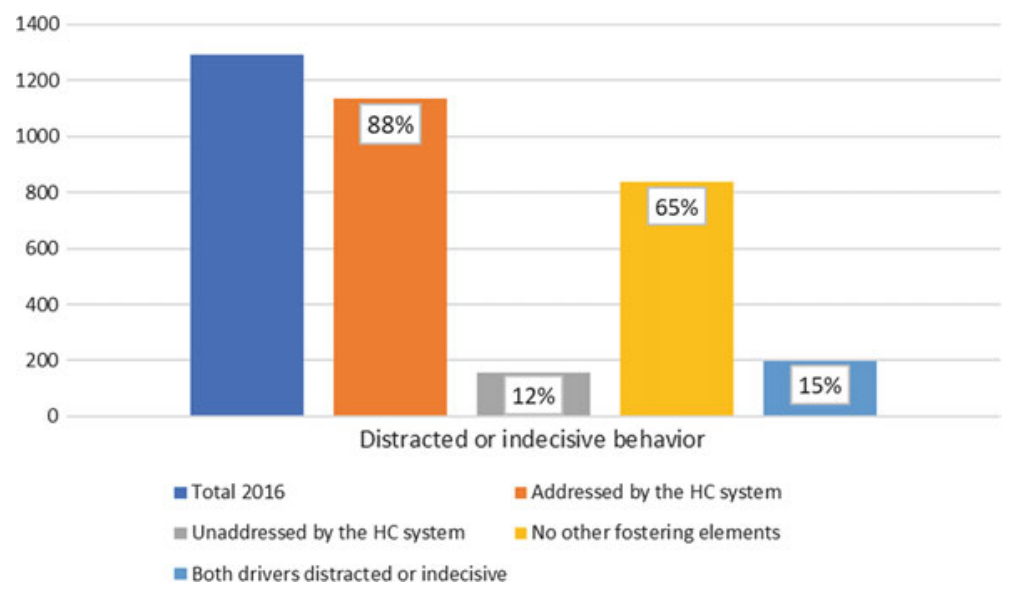

Fig. 7.3 Distracted driving or indecisive behavior

- 39 collisions occurred because the other driver was distracted or showed an indecisive behavior. Nevertheless, erasing the limited safety distance should make these crashes potentially avoidable or mitigated in their severity (especially because 31 of them were rear-end collisions).

- 36 collisions took place with the other vehicles because not keeping the safety distance. From the data available, it was not possible to determine the dynamics of these crashes, especially because it was not possible to assess how much the other vehicle involved concurred in the crash (i.e., insufficient safety distance). Therefore, adopting a conservative approach, all these crashes were judged as not addressable (or, to be more precise, they were not summed to the addressable ones).

Therefore, out of the 1,679 collisions caused or fostered by an insufficient safety distance, 1,643 (around 98\%) can be addressed by an L3 system such as the Highway Chauffeur, as long as the vehicle involved is an $\mathrm{HC}$ vehicle. The number of crashes addressable in this case is equal to around $26 \%$ of the 6,408 included in the ODD, (since the crashes considered in the analysis were those included in the ODD, no factor such as a wet road could have concurred to the event). Again, it should also be highlighted that, in the bullet list presented above, only some examples were reported and commented, while the same analysis was carried out for each one of the 1,291 scenarios recorded in ISTAT's database.

Speeding. If the Highway Chauffeur system is engaged, the driving speed cannot exceed the mandatory limit. Therefore, many of the 422 accidents occurred in scenarios falling within the ODD described above can be surely considered addressable. From an in-depth analysis, it appears that 


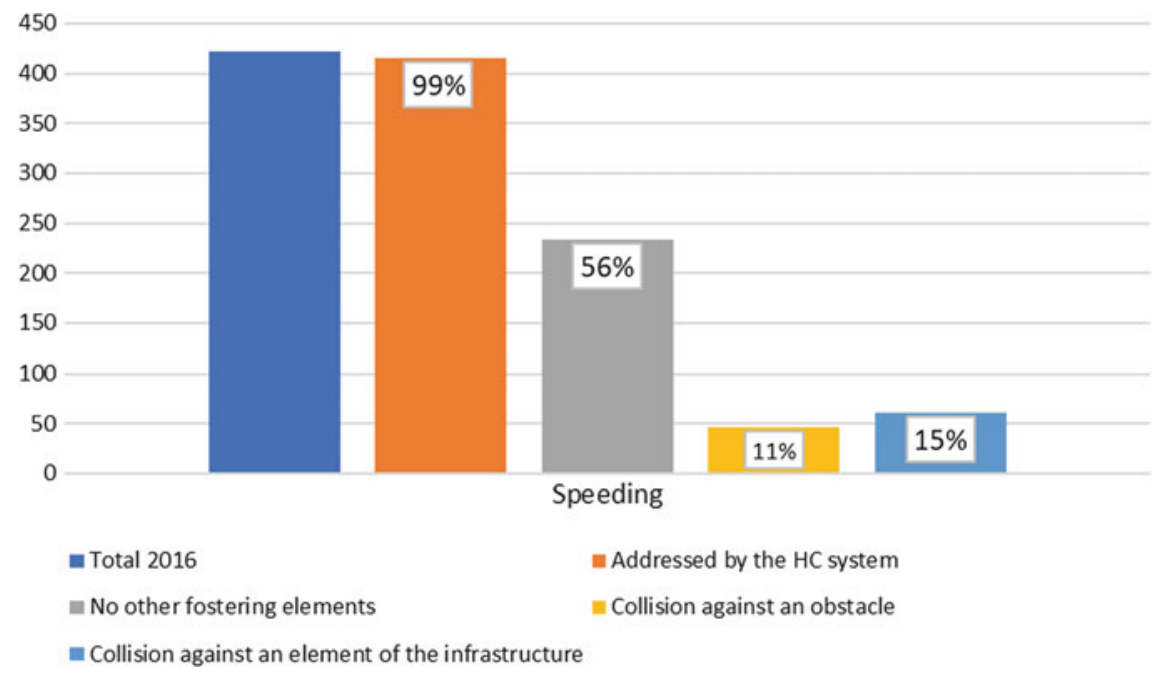

Fig. 7.4 Speeding

- 234 crashes occurred with no other fostering element apart from the speeding one, thus they are considered both addressable and most likely avoidable with an HC system.

-24 collisions took place while the driver of the other vehicle appeared distracted or showed an indecisive behavior. This type of accident was considered addressable, as well as the 16 that were rear-end collisions, since the speeding contribution can be prevented.

- 60 collisions occurred against an obstacle on the road. These accidents were considered addressable by the L3 system and also potentially preventable.

- 21 collisions occurred against a regularly stopped vehicle. These accidents were considered addressable by the L3 system and also potentially preventable.

- 61 collisions occurred against an element part of an infrastructure. Even these accidents were considered addressable by the L3 system and also potentially preventable when erasing the speeding factor. It should be noted that the crashes considered in the analysis are the ones included in the ODD, thus no factor such as a wet road can concur to the event.

Out of the 422 collisions caused or fostered by distraction or an indecisive behavior, 416 (around 99\%) resulted addressable and also presumably avoidable if one of the two vehicles involved is an HC. The number of crashes addressed in this case amounted to around $6.5 \%$ out of the 6,408 included in the ODD (Fig. 7.4).

Skidding or road departure due to distraction. As mentioned, having the system engaged should prevent crashes and events caused by a distracted behavior. 210 events occurred in the attempt to avoid an obstacle or a vehicle, presumably perceived by the human driver at the last moment. 75 events occurred without a recorded object to be 
avoided and, thus, were likely due to distraction alone. All these crashes (341 events) were considered addressable and likely preventable when the Highway Chauffeur is engaged. It is useful to remember that all these accidents happened in the intended ODD, thus with a dry road surface and no adhesion compromised (namely, with no other concurring factor in the dynamics of the event). This category represents $5 \%$ of the 6,408 events included in the ODD.

Skidding or road departure due to speeding. Also, in this case, the main external factors are the avoidance of an obstacle or a vehicle (297 events) and events not involving any object to be avoided (306 events). All these accidents (623 events) were considered addressable by the Highway Chauffeur system. Again, it is useful to remember that all these accidents occurred in the intended ODD, thus with a dry road surface and no adhesion compromised (namely, with no other concurring factor in the dynamics of the event). This category represents $10 \%$ of the 6,408 events included in the ODD.

Skidding or road departure to avoid an obstacle. These 155 events were not triggered by a vehicle's inappropriate behavior but still, while in its ODD, the HC vehicle should exploit its enhanced perception to reduce this type of event. Nevertheless, the dynamics of these crashes are not well defined by the data available and, therefore, were not considered addressable by the Highway Chauffeur system alone.

\subsection{Analysis of the Results}

The cases considered in Sect. 7.3 represent the more common ones, as far as causes and fostering elements are concerned, accounting alone for $70 \%$ of all the crashes occurred in scenarios ascribable to the ODD. It should also be considered that some crashes could not be analyzed in depth, such as the 431 cases in which no cause or fostering element is recorded for the vehicle and, thus, the dynamics of the crashes could not be recovered from the data available. Therefore, the share represented by the cases reported in the section above increases up to $76 \%$ of the analyzable events.

Around $4 \%$ of the crashes account for other scenarios, limited in numbers, that are not reported in this paper, not being statistically relevant (e.g., eight crashes in which the vehicle was overtaking without complying with the signaled overtaking ban were considered in the overall statistics, but not analyzed in depth in this paper). From the analysis of these cases, only 152 events $(\approx 0.02 \%)$ resulted as not addressable even with the automated system engaged. To obtain the most precise results possible, one last consideration should be made: 1,222 events occurred with the vehicle driving regularly, which means that the cause or the fostering element was probably due to the other vehicle involved. These events could have been avoided only with both vehicles equipped with the Highway Chauffeur system. Considering a market penetration of $10 \%$, the odds of avoidance are equal to $\approx 1 \%(0.009)$ which means that around 12 crashes of this category result addressable thanks to the use of $\mathrm{HC}$ vehicles on the Italian highways. The percentage of $0.9 \%$ should actually be applied to every type 
of crash in which the causes are ascribable to the other vehicle involved. However, in this paper, it was applied only when the numbers resulted statically relevant (the chosen threshold was set at 1,000 crashes).

The value of $10 \%$ for the market penetration parameter was chosen for the safety assessment in a short-medium term in order to retain a certain congruence with the 2016 accident-related data. Moreover, said parameter was chosen also because, in the long term, both the likely reached level and the capability of automated driving are hardly comparable to the Highway Chauffeur system. Besides, in the upcoming years, the impact on the safety of other technologies, such as C-ITS services and V2X communications, should be analyzed in their own capability of preventing accidents, but also in the possibilities granted by the joint implementation of L3 vehicles and cooperative technologies (e.g., the C-ITS Use Case Adverse Weather Conditions that can expand the ODD of a Highway Chauffeur vehicle or, at least, make the control transition from the automated system to the driver safer). This Take-Over transition can prove to be the cause or the fostering element for new types of crashes. However, it was not considered in this analysis, since it is not included in ISTAT's database for obvious reasons. Moreover, new types of crashes could and probably will arise from the complex relationship between the two authorities onboard: the human driver and the Highway Chauffeur system. This field of evaluation was not included in the results presented in this paper. With regard to this topic, an increasing number of works can be found in literature. However, numerical results referring to national realities are still missing and probably will continue to be missing until the system is used on public roads or a sufficient number of field tests are carried out.

\subsection{Conclusions and Future Works}

Out of the total number of crashes occurred during 2016, around $70 \%$ could have involved an $\mathrm{HC}$ vehicle driving in automated mode (meaning that $70 \%$ of the crashes occurred in the ODD conditions). This percentage could grow if the capability of the system to face light adverse weather conditions is taken into account. It is important to highlight how, considering the single event involving a single $\mathrm{HC}$ vehicle, the percentages of presumably avoidable crashes are rather high: $78 \%$ involving a distracted or an indecisive behavior, $98 \%$ involving an insufficient safety distance, and $99 \%$ when speeding is considered. It should be highlighted that the main impact is due to the incapability of the system to break traffic rules, thus abiding by safety distances and speed limits. However, it is also worth noticing how, in 2016, speeding and unsuitable safety distances accounted for around $33 \%$ of the crashes occurred in typical ODD scenarios. Moreover, driver distraction played a decisive role in $20 \%$ of the crashes considered in the analysis. Keeping these flawed behaviors out of the driving performances seems valuable on the basis of these percentages. Clearly, the analysis could be affected by the nature of the dataset considered, not intended for this type of evaluation. However, to prevent excessive uncertainties, each category of causes/fostering elements was analyzed on the basis of the type of crash. Where the 
pre-crash scenario was not clear, a conservative approach was adopted and no accident was considered as probably avoidable through the use of the Highway Chauffeur system. Besides, the analysis of the pre-crash scenario has already been employed for a first safety assessment of technologies not yet implemented on public roads [6, $14,15,21]$.

The estimated magnitude obtainable on safety is well represented by the total percentage of the addressed crashes (equal to 4,248), against the 6,408 considered in the ODD. The analysis highlighted that if each of the crashes occurred in 2016 had involved an $\mathrm{HC}$ vehicle, $66 \%$ of them could have been prevented or mitigated (on the basis of recorded causes and fostering elements). This represents the absolute number of crashes that, according to the analysis conducted, resulted to be very likely avoidable by an $\mathrm{HC}$ vehicle. In order to obtain an overall realistic estimation, this number must be projected considering the $10 \%$ market penetration assumed. Indeed, the greater number of crashes in the short term will still involve only traditional vehicles. Therefore, the estimated minimum number of crashes addressed through a $10 \%$ use of the Highway Chauffeur function on the Italian highways is equal to $6.6 \%$. This represents a lower limit that does not take into account the crashes for which the dynamics could not be determined by ISTAT's public database. It must also be highlighted that the majority of crashes in the scenario of a $10 \%$ market penetration would not involve an $\mathrm{HC}$ vehicle. Therefore, both results should be considered, that is, 66 and $6.6 \%$, each one with its statistical value.

To bluntly explicit the results obtained, if an effectiveness of $100 \%$ is considered, the owner of an $\mathrm{HC}$ vehicle would incur in at least $66 \%$ less crashes because the system can face successfully $66 \%$ of dangerous events when in the ODD. A traditional vehicle owner, in this scenario, would benefit from a crash reduction of $6.6 \%$, which is the percentage of crashes avoidable because the other vehicle involved is an $\mathrm{HC}$ vehicle.

Besides, it is interesting to note that the $66 \%$ result referring to the crashes addressable by an $\mathrm{HC}$ vehicle is similar to the $60 \%$ result obtained in [14], representing the crashes addressable by an autonomous vehicle (Fig. 7.5).

Future works could concern the completeness and inclusiveness of the data used for the evaluation, either accessing ISTAT's limited-access database or employing a totally different dataset in order to validate, verify or tune the results presented in this article. Besides, works capable of considering also the accident-related events occurred in adverse weather conditions could improve and enhance the output of this analysis. Different approaches could also be explored to determine the number of crashes ascribable to the typical ODD of a Highway Chauffeur system. For example, one approach could be to consider the field tests related to the statistical value of the activation time and/or $\mathrm{km}$ driven in automated mode derived and applied on specific highways. Another approach could involve the adoption of effectiveness assumptions, such as the ones reported in [17, 20,21] in order to avoid the $100 \%$ effectiveness hypothesis and obtain results closer to real outputs than to magnitude estimations. These coefficients should be obtained from field tests of vehicles achieving at least a level of automation equal to L2 or above, in order to consider the contribution of the sensors' suite and of software processing to the overall number 


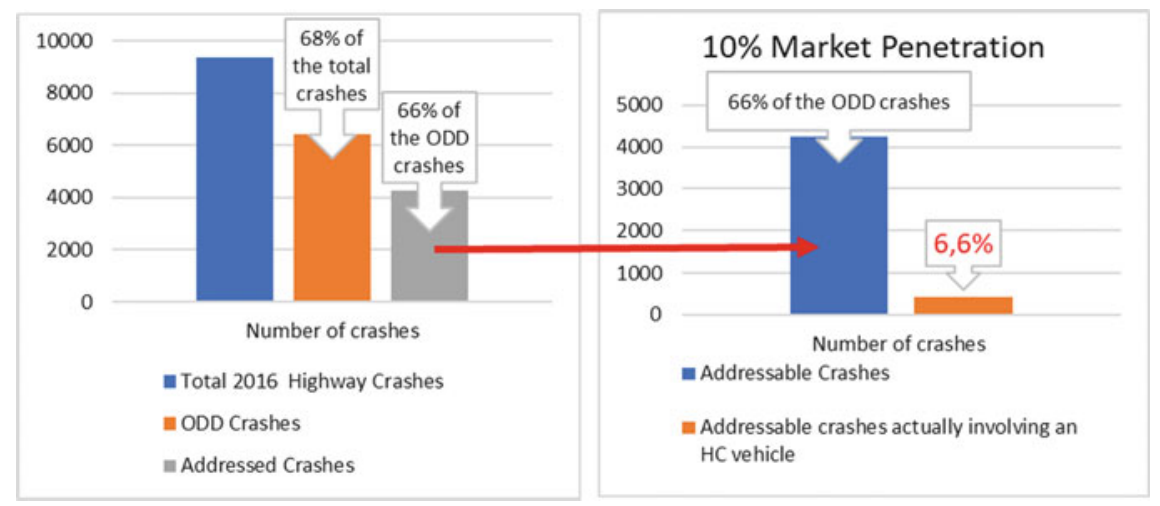

Fig. 7.5 Overall potential impact

of addressable crashes. In this paper, such approach was avoided because the field tests involving L3 systems on the Italian highways are not sufficient, therefore no effectiveness estimations could be found in literature. A step toward these results will be made within the C-Roads activities, during which $\mathrm{HC}$ vehicles will drive on the Italian highways and useful outputs will be obtained [22]. Besides, the performed analysis could be a useful input for cost-benefit analyses of the system in the short term. In this starting phase, in fact, there is the need to understand what the related benefit will be even though the implementation is not mature enough to use field tests data (both the prevention and the lowering of the consequences of a crash can be monetized and translated into economic benefits). As mentioned, the contribution of this paper indicates the magnitude of the safety impact obtainable from the use of the Highway Chauffeur system on the Italian highways. Therefore, future works in the same direction would be truly valuable to validate and tune the values obtained. Besides, the same analysis carried out on the Italian freeways would be valuable for an assessment in the medium term, in which it is foreseeable that also this type of road will be driven by a conditionally automated vehicle, as long as the ODD is met (the number of accidents on freeways in 2016 was equal to around 27,000 [23], against 9,360 on highways recorded by ISTAT). This kind of outputs could strongly improve the business case of an L3 system, even though not being exact results but rather estimations aimed at providing an order of magnitude. It is in fact clear that to state that a crash is avoidable on the basis of records should always be done with proper cautiousness, specifying that the objective of the work is not to determine what could have been avoided in the past, but rather to use the knowledge of the past to determine what will most likely be avoidable or at least addressable in the future. 


\section{References}

1. NHTSA and US Department of Transportation: Automated driving systems 2.0-A vision for safety. NHTSA National Highway Traffic Safety Administration, DOT HS 812 442, September, 2017. Retrieved from https://www.nhtsa.gov/manufacturers/automated-driving-systems, 10 April 2018

2. ERTRAC Working Group Connectivity and Automated Driving: Automated driving roadmap status: final for publication v.7.0.. ERTRAC European Road Transport Research Advisory Council, May 2017. Retrieved from http://www.ertrac.org/index.php?page=ertrac-roadmap, 18 Jan 2018

3. Pendleton, S.D., Andersen, H., Du, X., Shen, X., Meghjani, M., Eng, H.Y., Rus, D., Ang, H.M.: Perception, planning, control, and coordination for autonomous vehicles. Machines 5(1), 6 (2017)

4. C-Roads Platform, Working Group 2 Technical Aspects, Taskforce 2 Service Harmonization. Common C-ITS Service Definitions Other Hazardous Locations Notification (OHLN), version 1.07, February 7, 2018

5. ISTAT. http://www4.istat.it/it/archivio/87539

6. Rau, P., Yanagisawa, M., Najm, W.G.: Target crash population of automated vehicles. In: 24th International Technical Conference on the Enhanced Safety of Vehicles (ESV), No. 15-0430, pp. 1-11. National Highway Traffic Safety Administration, Washington, DC (2015)

7. Centre for Connected and Autonomous Vehicles: Market forecast for connected and autonomous vehicles. Transport Systems Catapult, Milton Keynes, MK, United Kingdom. July, 2017. Retrieved from https://www.gov.uk/government/publications/connected-andautonomous-vehicles-market-forcecast, 10 May 2018

8. AdaptIVe Project and A. Etemad: Deliverable D1.0 Final project result, June, 2017. Retrieved from https://www.adaptive-ip.eu/index.php/deliverables_papers.html, 17 Dec 2017

9. Suh, J., Kim, B., Yi, K.: Design and evaluation of a driving mode decision algorithm for automated driving vehicle on a motorway. IFAC-PapersOnLine 49(11), 115-120 (2016)

10. Katrakazas, C., Quddus, M., Chen, W., Deka, L.: Real-time motion planning methods for autonomous on-road driving: State-of-the-art and future research directions. Transp. Res. Part C: Emerg. Technol. 60, 416-442 (2015)

11. Best, A., Narang, S., Pasqualin, L., Barber, D., Manocha, D.: AutonoVi: autonomous vehicle planning with dynamic maneuvers and traffic constraints. In: IEEE/RSJ International Conference on Intelligent Robots and Systems (IROS). Vancouver, BC, Canada 24-28 Sept 2017

12. Jamson, A.H., Merat, N., Carsten, O.M.J., Lai, F.C.H.: Behavioural changes in drivers experiencing highly-automated vehicle control in varying traffic conditions. Transp. Res. Part C: Emerg. Technol. 30, 116-125 (2013)

13. Mcgehee, D.V., Brewer, M., Schwarz, C., Smith, B.W.: Review of automated vehicle technology: policy and implementation implications. In: IOWA Department of Transportation, DOT F 1700.7 (8-72), March 2016. https://rosap.ntl.bts.gov/view/dot/30702/dot_30702_DS1.pdf?

14. Eccles, K., Gross, F., Liu, M., Council, F.: Crash data analyses for vehicle-to-infrastructure communications for safety applications. US Department of Transportation, Report No. FHWAHRT-11-040, November 2012

15. Najm, G.W., Koopmann, J., Smith, D.J., Brewer, J.: Frequency of target crashes for intellidrive safety systems. US Department of Transportation, October 2010

16. Kulmala, R.: Ex-ante assessment of the safety effects of intelligent transport systems. Accid. Anal. Prev. 42, 1359-1369 (2010)

17. Yue, L., Abdel-Aty, M., Wu, Y., Wang, L.: Assessment of the safety benefits of vehicles advanced driver assistance, connectivity and low level automation systems. Accid. Anal. Prev. 117, 55-64 (2018)

18. Huisman, M.: Impacts of (cooperative) adaptive cruise control on traffic flow-a simulation case study on the effects of (cooperative) adaptive cruise control on the A15 highway. Delft University of Technology, 2016 
19. Hirz, M., Walzel, B.: Sensor and object recognition technologies for self-driving cars. Comput.Aided Des. Appl. 15(4), 501-508 (2018)

20. Li, T., Kockelman, K.M.: Valuing the safety benefits of connected and automated vehicle technologies. In: Transportation Research Board 95th Annual Meeting, 2016

21. Center for Transportation Research: Implications of connected and automated vehicles on the safety and operations of roadway networks: a final report. US Department of Transportation, Report No. FHWA/TX-16/0-6849-1, October 2016

22. Agriesti, S., Studer, L., Marchionni, G., Ponti, M., Gandini, P., Bresciani, C.: Highway chauffeur: state of the art and future evaluations-implementation scenarios and impact assessment. In: International Conference of Electrical and Electronic Technologies for Automotive, 2018

23. Li, T., Kockelman, K.M.: Valuing the safety benefits of connected and automated vehicle technologies. In: Transportation Research Board 95th Annual Meeting, 2016

Open Access This chapter is licensed under the terms of the Creative Commons Attribution 4.0 International License (http://creativecommons.org/licenses/by/4.0/), which permits use, sharing, adaptation, distribution and reproduction in any medium or format, as long as you give appropriate credit to the original author(s) and the source, provide a link to the Creative Commons license and indicate if changes were made.

The images or other third party material in this chapter are included in the chapter's Creative Commons license, unless indicated otherwise in a credit line to the material. If material is not included in the chapter's Creative Commons license and your intended use is not permitted by statutory regulation or exceeds the permitted use, you will need to obtain permission directly from the copyright holder.

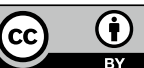

